\title{
Measurement of Mercury Distance, by Observing Transit of Mercury
}

\author{
Samaneh Shamyati ${ }^{1,2,3}$ \\ 1. A member of the Astronomy Society of IRAN, 15875-3671, Iran \\ 2. A member of Astronomy Center of Rey, Shahre Rey, Tehran, 1876943116, Iran \\ 3. Student of physics Payame noor University (PNU), Tehran, 19395-3697, Iran
}

\begin{abstract}
We will measure the distance between the Sun and the Moon by considering the Parallax and also by considering the Mercury Sun distance. The goal is to utilize the observed data. The way that we have used in this paper is based on two observational data in Iran (Mashhad $36^{\circ} 31^{\prime} \mathrm{N}, 59^{\circ} 48^{\prime} \mathrm{E}$ ) and Canada (Yukon Territory $69^{\circ} 27^{\prime} \mathrm{N}, 33^{\circ} 2^{\prime} \mathrm{E}$ ). It is required to mention that in Mercury transition 2016, I had personally collected the first place, Mashhad. In measuring the distance between Mercury and the Sun we have utilized the trigonometry terms and the related formulas. Using all the collected data and studying on the contacts of the mercury, has helped us to reach the best results.
\end{abstract}

Key words: Distance, Sun, Earth, Mercury, IRAN, Solar, Sun-Mercury, parallax.

\section{Nomenclature}

$\mathrm{R}_{\text {Earth }}=150015880=1000105867 \mathrm{Au}$

$\mathrm{R}_{\text {Mercury }}=65724412=0.438163746 \approx 0.43 \mathrm{Au}$

Most distance of the sun: $70 \times 10^{6}=0.46 \mathrm{Au}$

The average distance from sun: $58 \times 10^{6}=0.38 \mathrm{Au}$

The shortest distance to the sun: $46 \times 10^{6}=0.30 \mathrm{Au}$

$\mathrm{R}_{\mathrm{m}}=$ Distance between sun-mercury

$\mathrm{r}_{\mathrm{m}}=$ Radius of the Earth

$\triangle \mathrm{B}=$ parallax

$\mathrm{AB}=$ distance $($ IIRAN-CANADA $)$

$\alpha_{\mathrm{S}}=$ Solar paralax angle of observers AB

$\mathrm{R}_{\mathrm{e}}=$ Distance between sun- earth

\section{Introduction}

The start of this subject could go back to the year 1761, at which Edmond Halley had suggested a competition in the field of Mercury transition and Jin Nicolas Delicias collected some data then.

Corresponding author: Samaneh Shamyati, bachelor, research fields: astronomy, astrophysics (sun, aurora borealis) and cosmology.
Today the way is used for calculating the earth to Sun distance. In this paper both the Earth-Sun distance and Mercury-Sun distance have been used. The importance of this paper is due to the fact that we could use the observations to calculate the Mercury-Sun distance, using the Mercury transition. We could also confirm the previous measurements of the distance. The used formulas in this paper are all based on the results of working on this phenomenon and measuring the Sun-Earth distance. However the Mercury-Sun distance had been required.

\section{Calculation for the Distance of the Sun and Earth [4]:}

The point $\mathrm{O}$ indicated the center of the sun, $\mathrm{C}$ is the center of earth and $\mathrm{M}$ is the center of Mercury.

The observer in the point A, observes the Mercury's position $A^{\prime}$ on the surface of the sun. Likewise, the observer in the point B, observers the Mercury's position B' on the surface of the sun. Considering the theorem that: The measure of an exterior angle of a triangle is equal to the sum of the measures of the two interior angles that are not adjacent to it, for the two 
triangles BPM and APO:

$$
\begin{aligned}
\beta_{\mathrm{B}}+\alpha_{\mathrm{M}}=\rho_{1}, & \alpha_{\mathrm{S}}+\beta_{\mathrm{A}}=\rho_{2} \\
\rho_{2}=\rho_{1} & \rightarrow \beta_{\mathrm{B}}+\alpha_{\mathrm{M}}=\alpha_{\mathrm{S}}+\beta_{\mathrm{A}}
\end{aligned}
$$

The centers of Mercury, the Sun and Earth are placed on one line. Therefore:

$$
\alpha_{\mathrm{M}}-\alpha_{\mathrm{s}}=\beta_{\mathrm{A}}-\beta_{\mathrm{B}}
$$

where, $\Delta \beta$ is the Parallax, by simplification we will have:

$$
\begin{gathered}
\Delta \beta=\alpha_{\mathrm{s}}\left(\left(\frac{\alpha_{\mathrm{M}}}{\alpha_{\mathrm{s}}}\right)-1\right) \\
\tan \left(\frac{\alpha}{2}\right)=\frac{\mathrm{d} / 2}{\mathrm{D}}
\end{gathered}
$$

If $\alpha$ be a tiny angle therefore:

$$
\tan \left(\frac{\alpha}{2}\right)=\frac{1}{2} \tan \alpha
$$

By replacing them in the above equation, we have:

$$
\tan \alpha=\frac{\mathrm{d}}{\mathrm{D}}
$$

Considering the above expression and knowing that $R_{e}$ and $R_{M}$ are alternatively the Sun-earth distance and the Sun-Mercury distance, and that $\alpha_{\mathrm{s}}$ and $\alpha_{\mathrm{M}}$ are both tiny angles, we can use the below trigonometry relations in the two triangles $\mathrm{ABO}$ and $\mathrm{ABM}$.

$$
\alpha_{\mathrm{M}}=\frac{\mathrm{AB}}{\mathrm{R}_{\mathrm{e}}-\mathrm{R}_{\mathrm{M}}}, \alpha_{\mathrm{s}}=\frac{\mathrm{AB}}{\mathrm{R}_{\mathrm{e}}}
$$

Considering the above relations, we can calculate $\alpha_{\mathrm{s}}$ and $\alpha_{\mathrm{M}}$ :

$$
\frac{\alpha_{M}}{\alpha_{s}}=\frac{R_{e}}{R_{e}-R_{M}}
$$

By replacing it in the relation $\Delta \beta$, we will have:

$$
\left(\Delta \beta=\alpha_{\mathrm{s}}\left(\left(\frac{\mathrm{R}_{\mathrm{e}}}{\mathrm{R}_{\mathrm{e}}-\mathrm{R}_{\mathrm{M}}}\right)\right)-1\right)=\alpha_{\mathrm{s}}\left(\frac{\mathrm{R}_{\mathrm{M}}}{\mathrm{R}_{\mathrm{e}}-\mathrm{R}_{\mathrm{M}}}\right)
$$

Therefore:

$$
\left(\alpha_{\mathrm{s}}=\Delta \beta\left(\frac{\mathrm{R}_{\mathrm{e}}}{\mathrm{R}_{\mathrm{M}}}\right)-1\right) \quad \text { II }
$$

According to Fig. 2, $\alpha_{\mathrm{s}}$ would be the parallax of the Sun from the views of observers A and B. The ratio $\frac{R_{e}}{R_{M}}$ can be calculated via Kepler's third law (the Mercury orbital motion (a Mercury year: 87.979 days), an earth year lasts 365.25 days.

$$
\begin{gathered}
\left(\frac{R_{e}}{R_{M}}\right)^{3}=\left(\frac{T_{e}}{T_{M}}\right)^{2}, \\
\left(\frac{R_{e}}{R_{M}}\right)^{3}=\left(\frac{365.25}{87.969}\right)^{2} \Rightarrow \frac{R_{e}}{R_{M}}=2.583
\end{gathered}
$$

By replacing it in the expression (II), we have:

$\alpha_{s}=1.583 \Delta \beta$

Using the first relation, we can state $R_{e}$ in relation (I) like this:

$$
\mathrm{R}_{\mathrm{e}}=\frac{\mathrm{AB}}{\alpha_{\mathrm{s}}}=\frac{0.631 \mathrm{AB}}{\Delta \beta}
$$

As a result, to have $R_{e}$ we need the distance between the two observers (Iran-Canada) and $\Delta \beta$ of the observing data. Based on the observing data we have Table 1.

First by using trigonometry, we calculate the distance between Iran and Canada:

$$
\begin{aligned}
& \cos (\mathrm{C}-\mathrm{I})=\cos \left(90-\varphi_{\mathrm{c}}\right) \cos \left(90-\varphi_{\mathrm{I}}\right) \\
& +\sin \left(90-\varphi_{\mathrm{c}}\right) \sin \left(90-\varphi_{\mathrm{I}}\right) \cos \Delta \lambda \\
& \mathrm{IC}=\operatorname{Cos}^{-1} \theta \\
& \text { IC: (Distance of Iran to Canada) } \\
& \cos (\mathrm{C}-\mathrm{I})=\cos \left(90-\varphi_{\mathrm{c}}\right) \cos \left(90-\varphi_{\mathrm{I}}\right)+ \\
& \sin \left(90-\varphi_{\mathrm{c}}\right) \sin \left(90-\varphi_{\mathrm{I}}\right) \cos \Delta \lambda \\
& \cos \left(21^{\circ}\right) \cos \left(31^{\circ}\right)+\sin \left(21^{\circ}\right) \sin \left(31^{\circ}\right) \cos (74) \\
& =0.938(0.85)+0.35(0.51)(0.27) \\
& =0.79+0.048+0.838 \\
& \mathrm{IC}=\cos ^{-1}(0.838)=33.07 \\
& \overline{\mathrm{CI}}=\left(\cos ^{-1} \mathrm{x}^{\theta}\right) \\
& \overline{\mathrm{CI}}=\mathrm{R}_{\mathrm{e}} \cdot \theta \quad \theta: \mathrm{Rad} \\
& \mathrm{IC}=\mathrm{r}_{\mathrm{e}} \cdot \theta \Rightarrow \mathrm{IC}=(6371)(33.07)\left(\frac{\pi}{2}\right)= \\
& 9481003.65 \alpha_{\mathrm{s}}=\frac{9481003.65}{150000000}=0.0632 \rightarrow \alpha_{\mathrm{s}}=\frac{\mathrm{AB}}{\mathrm{R}_{\mathrm{e}}} \\
& \alpha_{\mathrm{M}}=\frac{9481003.65}{150000000-46000000}=0.091 \rightarrow \\
& \alpha_{\mathrm{M}}=\frac{\mathrm{AB}}{\mathrm{R}_{\mathrm{e}}-\mathrm{R}_{\mathrm{M}}}
\end{aligned}
$$

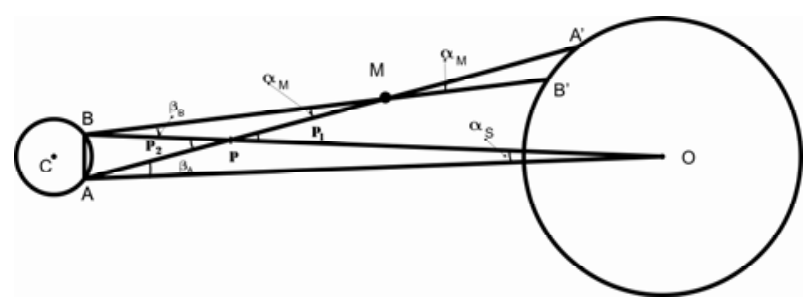

Fig. 1 Calculation for the distance of the Sun and Earth. 
Fig. 2 Calculation for the Angle of $\alpha$.

$\mathrm{d} / 2$

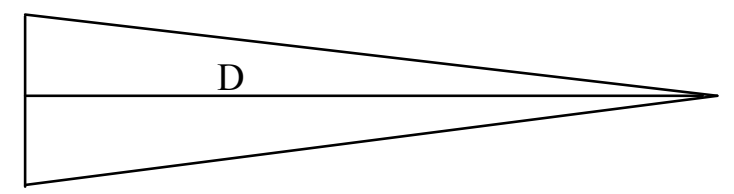

Table 1 Observation form (the transit of Mercury), Ahmad Nezam\&Samaneh shamyati, Ferdowsi University, Mashhad, Iran.

OBSERVATION FORM

The transit of Mercury

\begin{tabular}{|c|c|}
\hline \multicolumn{2}{|c|}{ Observatory } \\
\hline ObserverName:Ahmad Nezam & AssistanceName:MahsaMaram \\
\hline Longitude:E $59.4881^{\circ}$ & Latitude:N $36.3131^{\circ}$ \\
\hline Altitude:930 m & Place Name: ,Mashhad, Iran \\
\hline \multicolumn{2}{|c|}{ Email address:ahmad.nezam@gmail.com } \\
\hline \multicolumn{2}{|c|}{ Event Type } \\
\hline \multicolumn{2}{|c|}{ Transit: } \\
\hline \multicolumn{2}{|c|}{ Weather } \\
\hline Temperature $\left({ }^{\circ} \mathrm{C}\right): 23$ & Humidity (\%):- \\
\hline Percentage of Clear Sky (\%):80 & Visibility: \\
\hline \multicolumn{2}{|c|}{ Tools } \\
\hline Name of Telescope:TAL120 & Telescope Aperture:120 mm \\
\hline Focal length : $1000 \mathrm{~mm}$ & Telescope Mounting:Equatorial \\
\hline Telescope Eyepiece:00 mm & Optics: \\
\hline Drive : No & Filter:baaderastrsolar film \\
\hline \multicolumn{2}{|c|}{ Altitude sun } \\
\hline \multicolumn{2}{|c|}{ Sun Alt: $45^{\circ}$} \\
\hline \multicolumn{2}{|c|}{ Event Time \& Date } \\
\hline \multicolumn{2}{|l|}{ Year/ Month/ Day:2016/5/9 } \\
\hline \multicolumn{2}{|l|}{ One contact:(h/m/s)15:41:24 } \\
\hline \multicolumn{2}{|l|}{ Two contact: (h/m/s) 15:44:08 } \\
\hline \multicolumn{2}{|c|}{ Three contact: $(\mathrm{h} / \mathrm{m} / \mathrm{s})$ haven't been seen in Iran } \\
\hline \multicolumn{2}{|c|}{ Four contact: $(\mathrm{h} / \mathrm{m} / \mathrm{s})$ haven't been seen in Iran } \\
\hline \multicolumn{2}{|l|}{ Finally time: $(\mathrm{h} / \mathrm{m} / \mathrm{s})$ - } \\
\hline \multicolumn{2}{|c|}{ Timingmethod } \\
\hline Visual $\square$ & video recording \\
\hline $\mathrm{CCD}_{\square}$ & Photometry \\
\hline
\end{tabular}



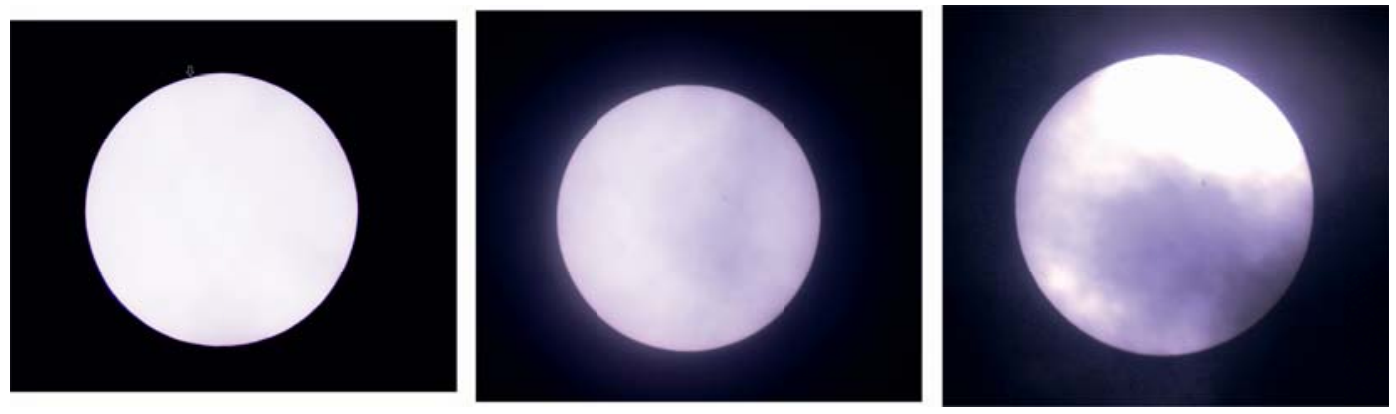

Fig. 3 photo by Ahmad Nezam, Observatory of Ferdowsi University, Mashhad, Iran.

Greatest Transit $=14: 57: 25.9$ UT1

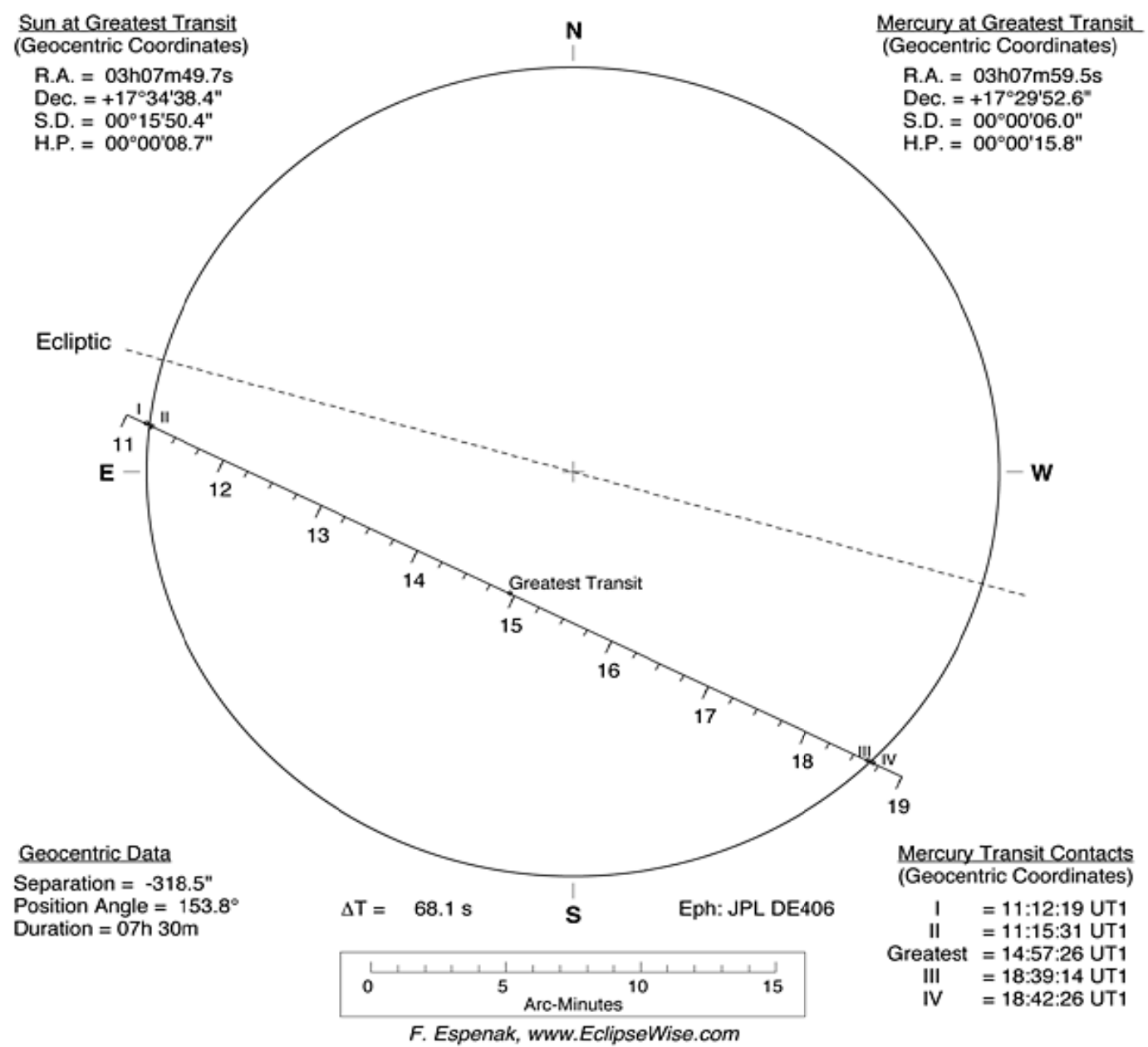

Fig. 4 Transit of Mercury :2016 May 09, F. Espenak, www.Eclipsewise.com.[1].

$$
\begin{aligned}
& \Delta \beta=\alpha_{\mathrm{s}}\left(\left(\frac{\alpha_{\mathrm{M}}}{\alpha_{\mathrm{s}}}\right)-1\right)=0.0632\left(\left(\frac{0.091}{0.0632}\right)-1\right) \\
& \left\{\begin{array}{l}
\stackrel{\Delta}{\mathrm{BMP}}: \beta_{\mathrm{B}}+\alpha_{\mathrm{M}}=\mathrm{P}_{1} \\
\stackrel{\Delta}{\mathrm{APO}}: \alpha_{\mathrm{S}}+\beta_{\mathrm{A}}=\mathrm{P}_{1}
\end{array} \Rightarrow \beta_{\mathrm{B}}+\alpha_{\mathrm{M}}=\alpha_{\mathrm{S}}+\beta_{\mathrm{A}}\right. \\
& =0.027 \\
& \mathrm{R}_{\mathrm{e}}=\frac{\mathrm{AB}}{\alpha_{\mathrm{s}}} \Rightarrow \mathrm{R}_{\mathrm{e}}=\frac{9481003.65}{0.0632}=150015880 \mathrm{mK} \\
& \Rightarrow \alpha_{\mathrm{M}}-\alpha_{\mathrm{S}}=\beta_{\mathrm{A}}-\beta_{\mathrm{B}}=\Delta \beta \\
& \Rightarrow \Delta \beta=\alpha_{M}-\alpha_{1}=\alpha_{M}\left(1-\frac{\alpha_{1}}{\alpha_{M}}\right) \quad \text { I }
\end{aligned}
$$




$$
\alpha=\frac{\mathrm{d}}{\mathrm{D}}
$$

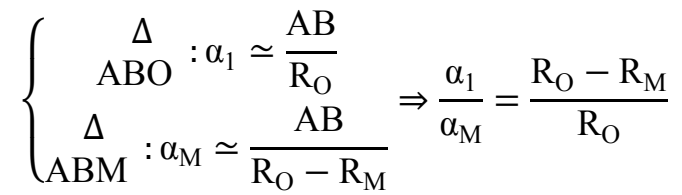

$$
\begin{aligned}
& =1-\frac{\mathrm{R}_{\mathrm{M}}}{\mathrm{R}_{\mathrm{S}}} \\
& \Rightarrow \quad \Delta \beta=\alpha_{\mathrm{M}}\left(1-1+\frac{\mathrm{R}_{\mathrm{M}}}{\mathrm{R}_{\mathrm{O}}}\right)=\alpha_{\mathrm{M}} \cdot \frac{\mathrm{R}_{\mathrm{M}}}{\mathrm{R}_{\mathrm{O}}} \quad \text { II }
\end{aligned}
$$

$$
\begin{aligned}
& \left(\frac{\mathrm{R}_{\mathrm{M}}}{\mathrm{R}_{\mathrm{O}}}\right)^{2}=\left(\frac{\mathrm{T}_{\mathrm{M}}}{\mathrm{EO}}\right)^{2}=\left(\frac{87.969}{365.25}\right)=(0.24)^{2} \Rightarrow \frac{\mathrm{R}_{\mathrm{M}}}{\mathrm{R}_{\mathrm{O}}}=0.24 \\
\mathrm{II} & \Rightarrow \quad \alpha_{\mathrm{M}}=\frac{\Delta \beta}{(0.24)}=4.17 \Delta \beta \Rightarrow \alpha_{\mathrm{M}} \\
& \Delta \beta=\alpha_{\mathrm{M}}(0.24) \Rightarrow \mathrm{\alpha}_{\mathrm{O}}-\mathrm{R}_{\mathrm{M}}=\frac{\Delta \beta}{4.17 \Delta \beta} \Rightarrow \mathrm{R}_{\mathrm{M}} \\
= & \frac{\Delta \beta}{\mathrm{R}_{\mathrm{O}}-\mathrm{R}_{\mathrm{M}}}=4.17 \Delta \beta \Rightarrow \mathrm{R}_{\mathrm{O}} \\
= & \mathrm{R}_{\mathrm{O}}-0.24 \frac{\mathrm{AB}}{\Delta \beta}
\end{aligned}
$$

\begin{tabular}{|c|c|c|c|c|c|c|c|c|c|c|}
\hline & $\begin{array}{l}\text { External } \\
\text { Ingress } \\
\text { h m s }\end{array}$ & $\begin{array}{l}\text { Sun } \\
\text { Alt } \\
0\end{array}$ & $\begin{array}{l}\text { Internal } \\
\text { Ingress } \\
\text { h m s }\end{array}$ & $\begin{array}{l}\text { Sun } \\
\text { Alt } \\
0\end{array}$ & $\begin{array}{c}\text { Greatest } \\
\text { Transit } \\
\text { h m s }\end{array}$ & $\begin{array}{l}\text { Sun } \\
\text { Alt } \\
0\end{array}$ & $\begin{array}{c}\text { Internal } \\
\text { Egress } \\
\text { h m s }\end{array}$ & $\begin{array}{l}\text { Sun } \\
\text { Alt } \\
0\end{array}$ & $\begin{array}{c}\text { External } \\
\text { Egress } \\
\text { h m s }\end{array}$ & $\begin{array}{l}\text { Sun } \\
\text { Alt } \\
0\end{array}$ \\
\hline \multicolumn{11}{|l|}{ Prince Ed.Is. } \\
\hline Charlottetown & $08: 13: 24$ & 24 & $08: 16: 36$ & 24 & $11: 57: 35$ & 58 & $15: 37: 57$ & 48 & $15: 41: 08$ & 48 \\
\hline \multicolumn{11}{|l|}{ Quebec } \\
\hline Beauport & $07: 13: 25$ & 18 & $07: 16: 37$ & 19 & $10: 57: 43$ & 54 & $14: 38: 04$ & 52 & $14: 41: 16$ & 52 \\
\hline Brossard & $07: 13: 27$ & 16 & $07: 16: 39$ & 17 & $10: 57: 47$ & 54 & $14: 38: 08$ & 54 & $14: 41: 19$ & 54 \\
\hline Cap Chat & $07: 13: 20$ & 21 & $07: 16: 32$ & 22 & $10: 57: 35$ & 54 & $14: 37: 59$ & 48 & $14: 41: 10$ & 48 \\
\hline Charlesbourg & $07: 13: 25$ & 18 & $07: 16: 37$ & 19 & $10: 57: 43$ & 54 & $14: 38: 04$ & 52 & $14: 41: 16$ & 52 \\
\hline Chicoutimi & $07: 13: 22$ & 18 & $07: 16: 34$ & 19 & $10: 57: 40$ & 53 & $14: 38: 03$ & 51 & $14: 41: 14$ & 51 \\
\hline Drummondville & $07: 13: 26$ & 17 & $07: 16: 39$ & 18 & $10: 57: 45$ & 54 & $14: 38: 06$ & 53 & $14: 41: 17$ & 53 \\
\hline Gatineau & $07: 13: 27$ & 15 & $07: 16: 39$ & 15 & $10: 57: 49$ & 52 & $14: 38: 10$ & 55 & $14: 41: 21$ & 55 \\
\hline Hull & $07: 13: 27$ & 15 & $07: 16: 40$ & 15 & $10: 57: 49$ & 52 & $14: 38: 10$ & 55 & $14: 41: 21$ & 55 \\
\hline La Salle & $07: 13: 27$ & 16 & $07: 16: 39$ & 17 & $10: 57: 47$ & 54 & $14: 38: 08$ & 54 & $14: 41: 19$ & 54 \\
\hline Laval & $07: 13: 27$ & 16 & $07: 16: 39$ & 17 & $10: 57: 47$ & 53 & $14: 38: 08$ & 54 & $14: 41: 19$ & 54 \\
\hline Longueuil & $07: 13: 27$ & 16 & $07: 16: 39$ & 17 & $10: 57: 47$ & 54 & $14: 38: 07$ & 54 & $14: 41: 19$ & 54 \\
\hline Montreal & $07: 13: 27$ & 16 & $07: 16: 39$ & 17 & $10: 57: 47$ & 54 & $14: 38: 08$ & 54 & $14: 41: 19$ & 54 \\
\hline Quebec & $07: 13: 25$ & 18 & $07: 16: 37$ & 19 & $10: 57: 43$ & 54 & $14: 38: 04$ & 52 & $14: 41: 16$ & 52 \\
\hline Sainte Foy & $07: 13: 25$ & 18 & $07: 16: 37$ & 19 & $10: 57: 43$ & 54 & $14: 38: 04$ & 52 & $14: 41: 16$ & 52 \\
\hline Saint Hubert & $07: 13: 27$ & 16 & $07: 16: 39$ & 17 & $10: 57: 47$ & 54 & $14: 38: 07$ & 54 & $14: 41: 19$ & 54 \\
\hline St. Laurent & $07: 13: 27$ & 16 & $07: 16: 39$ & 17 & $10: 57: 47$ & 53 & $14: 38: 08$ & 54 & $14: 41: 19$ & 54 \\
\hline St. Leonard & $07: 13: 27$ & 16 & $07: 16: 39$ & 17 & $10: 57: 47$ & 53 & $14: 38: 07$ & 54 & $14: 41: 19$ & 54 \\
\hline Shawinigan & $07: 13: 25$ & 17 & $07: 16: 38$ & 18 & $10: 57: 45$ & 53 & $14: 38: 06$ & 53 & $14: 41: 17$ & 53 \\
\hline Sherbrooke & $07: 13: 27$ & 17 & $07: 16: 39$ & 18 & $10: 57: 46$ & 54 & $14: 38: 06$ & 53 & $14: 41: 17$ & 53 \\
\hline Tr. Riviares & $07: 13: 26$ & 17 & $07: 16: 38$ & 18 & $10: 57: 45$ & 53 & $14: 38: 06$ & 53 & $14: 41: 17$ & 53 \\
\hline Verdun & $07: 13: 27$ & 16 & $07: 16: 39$ & 17 & $10: 57: 47$ & 54 & $14: 38: 08$ & 54 & $14: 41: 19$ & 54 \\
\hline \multicolumn{11}{|l|}{ Saskatchewan } \\
\hline Moose Jaw & -- & - & -- & - & $08: 57: 59$ & 32 & $12: 38: 33$ & 57 & $12: 41: 44$ & 57 \\
\hline Regina & -- & - & $05: 16: 24$ & -1 & $08: 57: 58$ & 33 & $12: 38: 32$ & 57 & $12: 41: 43$ & 57 \\
\hline Saskatoon & -- & - & $05: 16: 20$ & -1 & $08: 57: 56$ & 31 & $12: 38: 31$ & 55 & $12: 41: 43$ & 55 \\
\hline \multicolumn{11}{|l|}{$\begin{array}{l}\text { Yukon } \\
\text { Territory }\end{array}$} \\
\hline Dawson & -- & - & -- & - & $07: 57: 30$ & 14 & $11: 38: 33$ & 37 & $11: 41: 45$ & 37 \\
\hline Whitehorse & -- & - & -- & - & $07: 57: 38$ & 15 & $11: 38: 37$ & 40 & $11: 41: 48$ & 41 \\
\hline
\end{tabular}

Table 2 Canada (PEI-Yukon Territory).[5].

Local Circumstances for Transit of Mercury of 2016 May 09

"2016 Transit of Mercury", Fred Espenak, Observer's Handbook 2016, Royal Astronomical Society of Canada 
Table 3 Geocentric phases of the 2016 transit of Mercury. [2, 3].

\begin{tabular}{|c|c|c|}
\hline Event & Universal Time & Position Angle \\
\hline Contact I & $11: 12: 19$ & $83.2^{\circ}$ \\
\hline Contact II & $11: 15: 31$ & $83.5^{\circ}$ \\
\hline Greatest Transit & $14: 57: 26$ & $153.8^{\circ}$ \\
\hline Contact III & $18: 39: 14$ & $224.1^{\circ}$ \\
\hline Contact IV & $18: 42: 26$ & $224.4^{\circ}$ \\
\hline
\end{tabular}

\section{Results and Discussion}

Now we should replace the numbers in the equations. Therefore, we would have the distance between the Sun and Earth. Then according to Fig. 1 and calculation via Spherical trigonometry, we can measure the distance between the Sun and Mercury.

$$
\begin{aligned}
\mathrm{R}_{\mathrm{M}}=150000000 & -0.24 \frac{9481003.65}{0.027} \\
= & 150000000-84275588 \\
= & 65724412=0.4 \mathrm{Au}
\end{aligned}
$$

\section{Acknowledgement}

Special thanks to: Dr. Mehdi Daneshyar (the chairman of Shahrerey Astronomy Center); Dr. Atila pro (head of the Middle East IOTA); Dr. Ebadeh Moradi (a professor in Farhangiyan University);
Ehsan Mehrjoo (Astronomy \& Astrophysics Olympiad Teacher); Fatemeh Hematian (language translator); Shahla Shoja-Amineh (amateur astronomer); Ahmed Nezam (amateur astronomer).

\section{References}

[1] Explanatory Supplement to the Astronomical Ephemerise and the American Ephemeris and Nautical Almanac, 1974, Her Majesty' Nautical Almanac Office, London.

[2] Espenak, F. 2015. Six Millennium Canon of Transits: -2999 to +3000. Portal, AZ: Astropixels Publishing.

[3] Meeus, J. 1989. Transits. Richmond, VA: Willmann-Bell, Inc.

[4] Hashemi-Niari, F. 2016. "Calculate the Distance from Earth to the Sun by Observing Transit." Astronomy Magazine 5: 23.

[5] Espenak, F. 2016. 2016 Transit of Mercury. Observer's Handbook 2016, Royal Astronomical Society of Canada. 